\title{
Estados emocionales y nivel de actividad física Len la Escuela Militar de Cadetes General José María Córdova
}

Emotional States and the Level of Physical Activity in the Military Academy [Escuela Militar de Cadetes General José María Córdova]

(c) (i)

Claudia Xiomara Santos León Sergio Mauricio Castañeda Tovar Ninosca Sofia Caiaffa Bermúdez Oscar Mauricio Guzmán Garzón

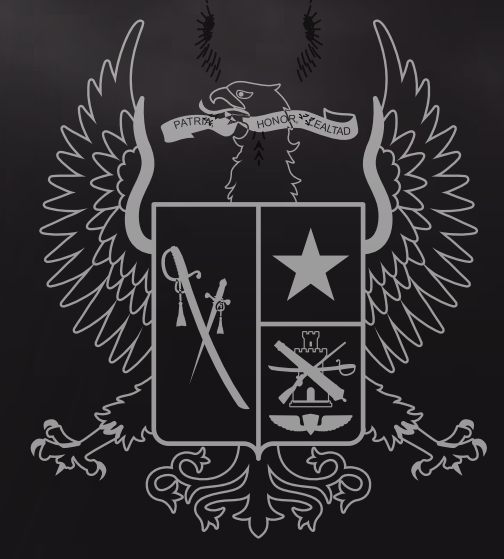


ISSN-I: 2011-7197 | e-ISSN: 2463-2236 Publicación Semestral

ID: 2011-7191.MCT.11103

Title: $\quad$ Emotional States and the Level of Physical Activity in the Military Academy

Subtitle: [Escuela Militar de Cadetes Genera José María Córdova]

Título: $\quad$ Estados emocionales y nivel de actividad física en la Escuela Militar de Cadetes General José María Córdova

Author (s) / Autor (es):

Santos León, Castañeda Tovar, Caiaffa Bermúdez, \& Guzmán Garzón

Keywords / Palabras Clave:

[en]

physical activity; anxiety; depression; body mass index; military

[es] actividad física; ansiedad; depresión; índice de masa corporal; militar

Submited: 2017-02-20

Acepted: 2017-03-05

\section{Resumen}

Introducción: la proyección integral del militar exige que sean tenidas en cuenta habilidades, aptitudes, competencias, condiciones físicas y psicológicas, entre otras, para garantizar un buen desempeño

operacional. Por esto es importante estudiar, desde el campo del movimiento corporal humano, aspectos como los estados emocionales y el rendimiento físico de quienes se preparan para la vida militar. Objetivo analizar los estados emocionales (ansiedad/depresión) y el nivel de actividad física, y sus posibles relaciones, de los estudiantes de la Escuela Militar que presentan peso corporal normal y que tienen sobrepeso.

Metodología: se realizó un estudio cuantitativo, descriptivo, y con componente analítico, con el cual se valoraron 76 estudiantes de sexo masculino y femenino. Las características de la población señalan que su edad promedio es $19.0 \pm 1.7$ años, la talla evidencia valores sobre $1.70 \pm 3.2 \mathrm{~cm}$., el peso tiene valores de $74.50 \pm 2.1 \mathrm{~kg}$., y, según la clasificación para el Índice de Masa Corporal (IMC), se muestra un resultado de $25.90 \pm 0.50 \mathrm{Kg} / \mathrm{m} 2$. Resultados: en la variable de estados emocionales, la ansiedad estado reveló una mediana de 29.57\%; en la de depresión, la categoría predominante en la muestra fue depresión mínima; en el nivel de la actividad física, se destaca el componente vigoroso con valores de

7.200 MET-minuto/semana, según los criterios del

IPAQ. Conclusión: se encontró una correlación entre la ansiedad-estado y ansiedad rasgo ( $r 2=0.52)$. Al aplicar

pruebas no paramétricas se pudo establecer que,

a mayor categoría de depresión, mayor puntaje de ansiedad-estado, en la muestra estudiada $p<0.05$. En cuanto a la comparación entre las variables de estados

emocionales y las de actividad física del estudio,

inicialmente la variable de actividad física vigorosa, comparada con las categorías de depresión, parecía reflejar cierta diferencia importante; sin embargo, al realizar pruebas no paramétricas, se observó que

todas las variables MET fueron estadísticamente iguales para las categorías de depresión. Esto indica que no hay influencia entre estas y el nivel de actividad física, no tiene relación con el grado de depresión que se experimente.

\section{Abstract}

Introduction: the integral projection of the military requires that skills, aptitudes, competencies, physical and psychological conditions, among others, be taken into account in order to guarantee a good operational performance. This is why it is important to study, from the field of

human body movement, aspects such as the emotional states and the physical performance of those who prepare for military life. Objective: to analyze the emotional states (anxiety / depression) and the level of physical activity, and its possible relationships, of the

students of the Military School who present normal body weight and who are overweight. Methodology: a quantitative, descriptive, and analytical component study was conducted. with which 76 male and female students were assessed. The characteristics of the population indicate that their average age is $19.0+1.7$ years, the size evidences values over $1.70 \pm 3.2 \mathrm{~cm}$., The weight has values of 74.50 $\pm 2.1 \mathrm{~kg}$., and according to the classification for the Body Mass Index (BMI), a result of $25.90 \pm 0.50 \mathrm{Kg} / \mathrm{m} 2$ is shown. Results: in the variable of emotional states, the anxiety-state revealed a median of $29.57 \%$; in the case of depression, the predominant category in the sample was minimal depression; In the level of physical activity, the vigorous component stands out with values of 7,200 MET-minute / week, according to the criteria of the IPAQ. Conclusion: a correlation between anxiety-state and trait anxiety was found (r2 = 0.52). When applying nonparametric tests, it was possible to establish that the higher the category of depression, the higher the anxiety-state score, in the sample studied $p<0.05$. As for the comparison between the

variables of emotional states and those of physical activity of the study, initially

the variable of vigorous physical activity, compared with the categories of depression, seemed to reflect some important difference; however, when performing nonparametric tests, it was observed that all MET variables were statistically equal for the depression categories. This indicates that there is no influence between these and the level of physical activity; it is not related to the degree of depression that is experienced.

\section{Claudia Xiomara Santos León, Ps sp}

Bio:

Posgraduada en Logoterapia y Análisis Existencial de la Conducta, Colectivo Aquí y Ahora en convenio con la Universidad de Flores en Argentina Especialista en Dirección de Talento Humano, Universidad Sergio Arboleda Psicóloga, Universidad de los Andes

Ciudad:

Bogotá D.C. [co]

\section{Ninosca Sofia Caiaffa Bermúdez, BA}

Bio:

Nutricionista Dietista, Universidad del Atlántico.

Antropometrista certificate Nivel II en Cineantropometría, ISAK, (The International Society for the Advancement of Kineanthropometry)

Ciudad:

Bogotá D.C. [co]
Lic Sergio Mauricio Castañeda Tovar, MSc

Bio:

Magíster en Pedagogía de la Cultura Física, Universidad Pedagógica y

Tecnológica de Colombia (UPTC)

Licenciado en Educación Física, Universidad Pedagógica Nacional

Ciudad:

Bogotá D.C. [co].

Oscar Mauricio Guzmán Garzón, MA

Magister en Actividad Física para la Salud, Universidad Santo Tomas. Profesional en Cultura Física

Ciudad:

Bogotá D.C. [co] 


\section{Estados Emocionales y Nivel de Actividad Física en la Escuela Militar General José María Córdova}

Claudia Xiomara Santos León Sergio Mauricio Castañeda Tovar Ninosca Sofia Caiaffa Bermúdez Oscar Mauricio Guzmán Garzón

La Escuela Militar de Cadetes General José María Córdova es una institución que tiene la misión de realizar la formación de los futuros oficiales del Ejército de Colombia. En esta empresa, la proyección integral del militar exige que sean tenidos en cuenta conocimientos, habilidades, aptitudes, competencias, condiciones físicas y psicológicas, entre otros, para garantizar un buen desempeño operacional. Es así como se ve la importancia de estudiar, desde el campo del movimiento corporal humano, aspectos como los estados emocionales y el rendimiento físico de quienes se preparan para la vida militar.

En cuanto a los estados emocionales, se observa que la ansiedad y la depresión, en el contexto militar, han sido predominantemente asociadas a situaciones de combate y lesiones causadas por las minas antipersonales o eventos experimentados en el desarrollo del conflicto armado. De acuerdo con lo planteado por Loaiza y Posada (2016) en Colombia las investigaciones con militares han girado en torno a estrés postraumático causado por tomas guerrilleras, secuestro, carros bomba, ataques por sorpresa, etc., situaciones relacionadas con altos niveles de estrés y de trauma. También se han realizado estudios de salud mental en oficiales de la fuerza aérea y las implicaciones psicológicas que ha tenido el conflicto armado.

En primer lugar, es posible encontrar un estudio como el realizado por Pérez Hidalgo y Rodríguez González (2011) en el que se utiliza el cuestionario de ansiedad-estado/rasgo para analizar los estados emocionales de una unidad del Ejército Español en la zona de operaciones, aplicando el instrumento antes y después del despliegue. En este estudio se encontraron diferencias significativas entre las medidas de ansiedad-estado, en primera y segunda fase, condicionadas por el sexo y el nivel académico. Aunque los niveles de ansiedad-estado y ansiedad-rasgo no alcanzaron en ningún momento puntuaciones de valores negativos, concluyeron que los niveles formativos superiores favorecen un afrontamiento positivo de la experiencia. 


\section{Estado de Ansiedad}

La ansiedad se considera como una reacción humana común que surge como respuesta adaptativa de los individuos frente a una condición que evalúan como perjudicial para su propia existencia. Pero, cuando esta respuesta emocional se torna demasiado intensa y reduce la eficacia personal, o la satisfacción con la vida, en situaciones que no revisten un peligro real, es posible hablar de los trastornos de ansiedad. Generalmente, el término ansiedad se define como un sentimiento de miedo y aprensión difuso, vago y muy desagradable. Además, este sentimiento presenta combinaciones de los siguientes síntomas: ritmo cardiaco acelerado, respiración entrecortada, diarrea, pérdida del apetito, desmayos, mareos, sudoración, insomnio, micción frecuente y estremecimientos (Sarason \& Sarason, 2006). En cuanto al estado de ánimo deprimido, es el síntoma más notable de la depresión. Las personas depresivas experimentan síntomas de tristeza, desaliento y una aflicción excesiva y prolongada; en ellas son comunes los sentimientos de inutilidad y de pérdida de la alegría de vivir, incluso puede ocurrir llanto violento como una reacción general de frustración y la cólera, sin que estos accesos estén relacionados de manera directa con una situación estresante específica. (Córdoba, 2011)

En el contexto de las escuelas de formación militar, en el que la población que ingresa es joven, la información sobre ansiedad y depresión es escasa. Esto posiblemente se debe a que en el proceso de admisión a la carrera de ciencias militares se realizan pruebas psicológicas que garantizan un perfil sano a nivel clínico. Sin embargo, es posible que puedan aparecer perturbaciones psicológicas un tiempo después del inicio de su vida profesional y militar una vez que se enfrentan situaciones demandantes a nivel psicológico que pueden exceder su capacidad de respuesta.

Ahora bien, en cuanto a la actividad física, la Organización Mundial de la Salud, OMS, en su estrategia "Salud para todos" (2010), incluye entre sus objetivos la reducción de la prevalencia de sobrepeso y obesidad en todos los grupos de edad, así como el incremento de la proporción de adultos que realiza actividad física moderada diaria. (Borba Neves, 2008). Es así como un alto nivel de condición física está relacionado directamente con un grado óptimo de respuesta fisiológica. (Bustamante, y otros, 2011). En este sentido, se destaca que una preparación física acorde al desarrollo de su labor y una equilibrada composición corporal le permitirán al militar contar con niveles altos de rendimiento físico para el desarrollo de diferentes operaciones. (Castañeda Tovar \& Caiaffa Bermúdez, 2015)

De los estudios realizados, ha quedado demostrado cómo la realización de actividad física, de acuerdo a unos criterios de individualidad, tipo, duración, intensidad, frecuencia y progresión, (Heyward, 1996), mejora la salud de la persona y ayuda al tratamiento de enfermedades crónicas no transmisibles.

Asimismo, se identificaron estudios afines con la obesidad, sobrepeso y el peso normal como los realizados por Sánchez Daza, Herrera González y Daza Mora (2012) en los cuales se evaluó el riesgo cardiovascular en aviadores militares del Ejército Colombiano (Borba Neves, 2008). Estos estudiaron la prevalencia de sobrepeso y obesidad en militares del Ejército Brasilero. Por otro lado, encontramos escasos antecedentes de estudios realizados en el contexto militar relacionados con la ansiedad y la depresión.

Es así como se ve la importancia de analizar los estados emocionales (ansiedad/depresión) y el nivel de actividad física, y sus posibles relaciones, de los estudiantes de la Escuela Militar que presentan peso corporal normal y que tienen sobrepeso.

\section{Metodología}

La investigación tiene un enfoque cuantitativo, en donde se realizó un estudio de tipo observacional descriptivo, con componente analítico, tomando datos de fuentes de información primarias. En este se analizaron los estados emocionales (depresión y ansiedad) y el nivel de actividad física de dos grupos de cadetes de la ESMIC que fueron conformados a partir del criterio de Índice de Masa Corporal (IMC).

El diseño de las muestras fue realizado por conveniencia, ya que de la población total de estudiantes se escogieron 76 cadetes (hombres y mujeres) de la ESMIC que cursaban entre 1 y 6 semestre proporcionalmente, y con edades comprendidas entre los 17 y los 21 años de edad, procedentes de diversas partes de Colombia. Estos cadetes, se clasificaron entre un IMC normal y con sobrepeso.

Se segmentó la muestra en dos grupos: el grupo 1 (G1) estuvo conformado por $\mathbf{2 5}$ cadetes, entre hombres y mujeres, cuyo IMC estaba en el rango de peso normal (entre 18.5 y $24.9 \mathrm{~kg} / \mathrm{m} 2$ ), y el grupo 2 (G2), estuvo conformado por $\mathbf{5 1}$ cadetes, entre hombres y mujeres, cuyo IMC estaba en el rango de sobrepeso (entre 25,0 y 29,9kg/m2), siguiendo los parámetros de clasificación del IMC establecido por la organización mundial de la salud.

Dentro de los criterios de inclusión se tuvieron en cuenta que los cadetes fueran estudiantes de la institución, y que además hayan tenido la valoración del nutricionista en el primer semestre del año 2015, como parte del desarrollo del programa de cultura física aplicado por la misma entidad en el Centro de Investigación de la Escuela Militar.

Los criterios de exclusión tenidos en cuenta fueron que el estudiante no tuviera antecedentes psiquiátricos, y que se no viera vinculado a investigaciones disciplinarias internas durante el proceso.

La valoración nutricional se realizó a partir de la toma de medidas antropométricas tales como el peso y la talla, siguiendo las normas o metodología de referencia en cineantropometría, basada en el protocolo de la ISAK (International Society for Advancement in Kineanthropometry). (ISAK, 2001)

Para la toma del peso se utilizó una báscula electrónica de peso corporal (Seca 813, capacidad de 150 kg, precisión de0.1kg). La talla fue medida en un estadiómetro portátil con una precisión de $0.1 \mathrm{~cm}$. Estas medidas se le tomaron a los sujetos sin zapatos ni medias, y con pantaloneta y camiseta. La estatura se tomó con los pies y las rodillas juntas; los talones, la cara posterior de los glúteos y la cabeza bien adheridos al plano del tallímetro. Así, con la ayuda de una pieza deslizante que desciende hasta el vertex de la cabeza, se leyó el valor de la talla en centímetros. El Índice de Masa Corporal (IMC), se calculó bajo los parámetros establecidos por la OMS, a partir de la fórmula de peso/talla 2 expresado en $\mathrm{kg} / \mathrm{m} 2$, clasificando a los individuos en bajo peso, normal, sobrepeso y obeso.

Para evaluar la ansiedad se utilizó la escala de ansiedadestado/rasgo (STAI) de Spielberger (Spielberger, Gorsuch, \& Lushene, 2011). El decatipo registrado en la puntuación de cada participante del estudio permitía su distribución de modo tal que se establecieron las diferentes categorías.

Para el análisis de la depresión se utilizó el inventario para la depresión de Beck BDIII. El cuestionario se aplicó de forma colectiva, se dieron las respectivas instrucciones y se procedió a la calificación 
determinando la puntuación directa, para posteriormente clasificar la severidad de la depresión en: mínima de $\mathbf{0}$ a $\mathbf{1 3}$ puntos, leve de $\mathbf{1 4}$ a $\mathbf{1 9}$ puntos, moderada entre $\mathbf{2 0}$ y $\mathbf{2 8}$ puntos y grave de $\mathbf{2 9}$ a $\mathbf{6 3}$ puntos. (Sanz Fernández, Vázquez Valverde, \& Luis Perdigón, 2003)

Los estados emocionales (ansiedad y depresión) se dividieron en variables discretas de la siguiente manera: la ansiedad, en tipo de ansiedad-estado y tipo de ansiedad-rasgo. Cada una de estas fue valorada a su vez en puntaje directo, decatipo, percentil; clasificadas en las categorías emocionalmente estable, mínima molestia de ansiedad, y presencia de síntomas de ansiedad-estado y/o rasgo.

El instrumento de evaluación en cuanto a la condición física fue la versión corta del IPAQ (Craig, y otros, 2003), que expresa los resultados de gasto calórico en MET en los tres dominios: andar, actividad moderada, actividad vigorosa.

El análisis estadístico se realizó por medio del programa estadístico $\mathbf{R}$ versión 3.2.5, destacando las medidas de tendencia central y de dispersión, y se aplicó la prueba no paramétrica Kruskalwallis.

\section{Resultados}

A continuación, se presentan los resultados de las variables estudiadas (estados emocionales de ansiedad/depresión, nivel de actividad física y peso corporal), y su correlación.

En la Gráfica 1 se muestra que la categoría de mínima de depresión es la que predomina cuando se es emocionalmente estable, cuando se tienen pocas molestias de ansiedad o hay presencia de síntomas de ansiedad-estado o ansiedad-rasgo. En el caso de experimentarse alguna molestia de ansiedad o síntomas de ansiedad-estado, hay ocasionalmente sensación de depresión leve, pero es reducido que se perciba depresión moderada.

En la Gráfica 2 se puede apreciar cómo a medida que aumenta la presencia de ansiedad-estado, la depresión es mayor.

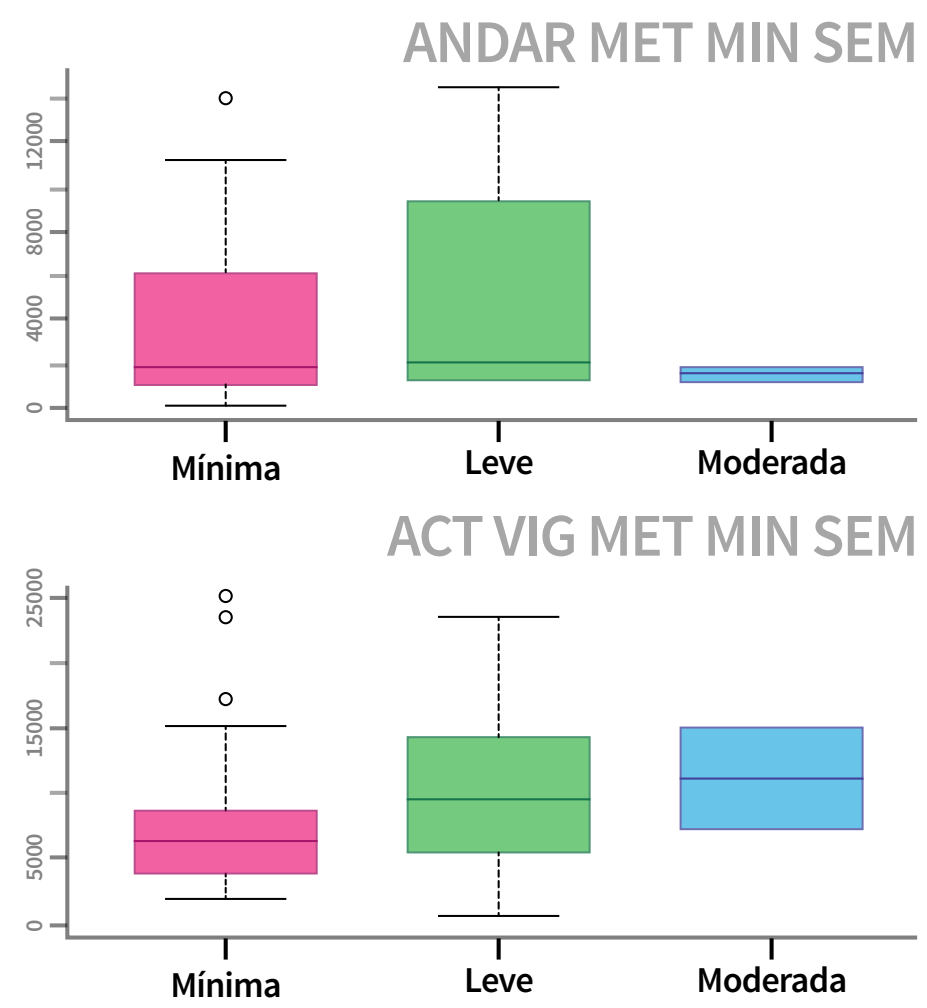

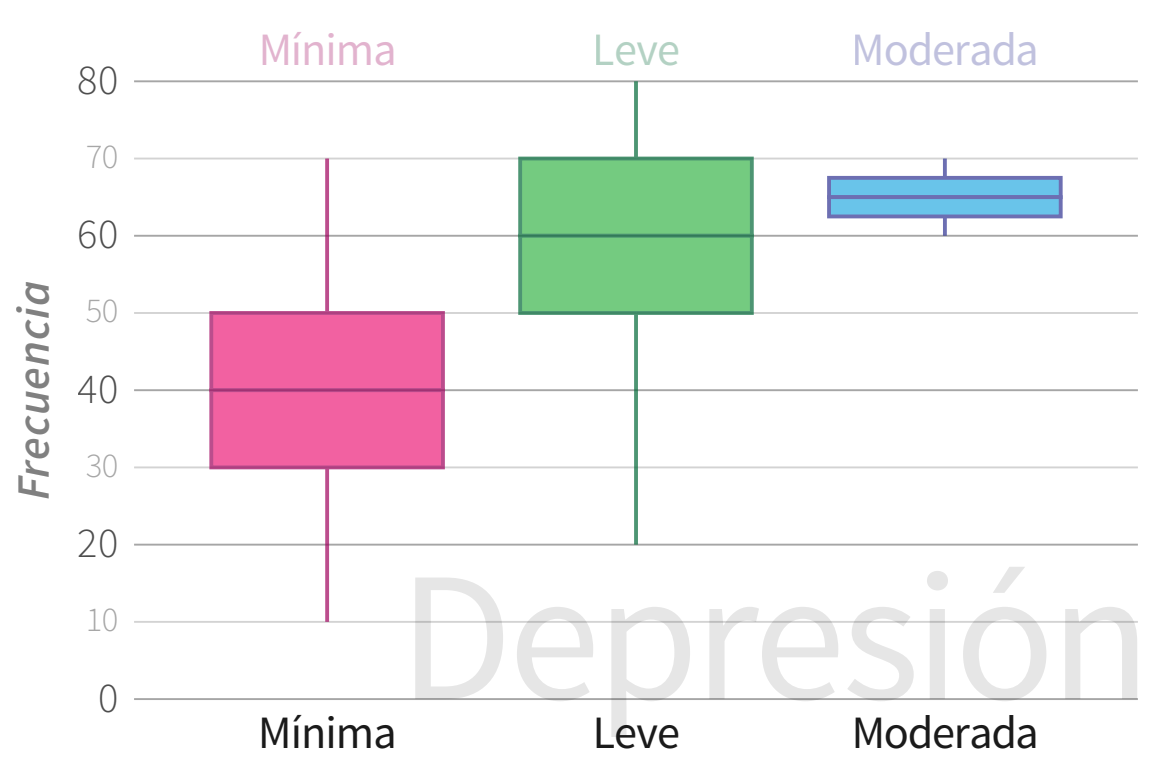

Gráfica 1 Perfiles de ansiedad comparado con la categoría de depresión

Fuente: Elaboración Propia

En la Tabla 1 (siguiente) se describe el cruce de las variables ansiedad-estado y depresión mediante pruebas no paramétricas, en el que se encuentra un valor $p$ con significancia $p=0.00$

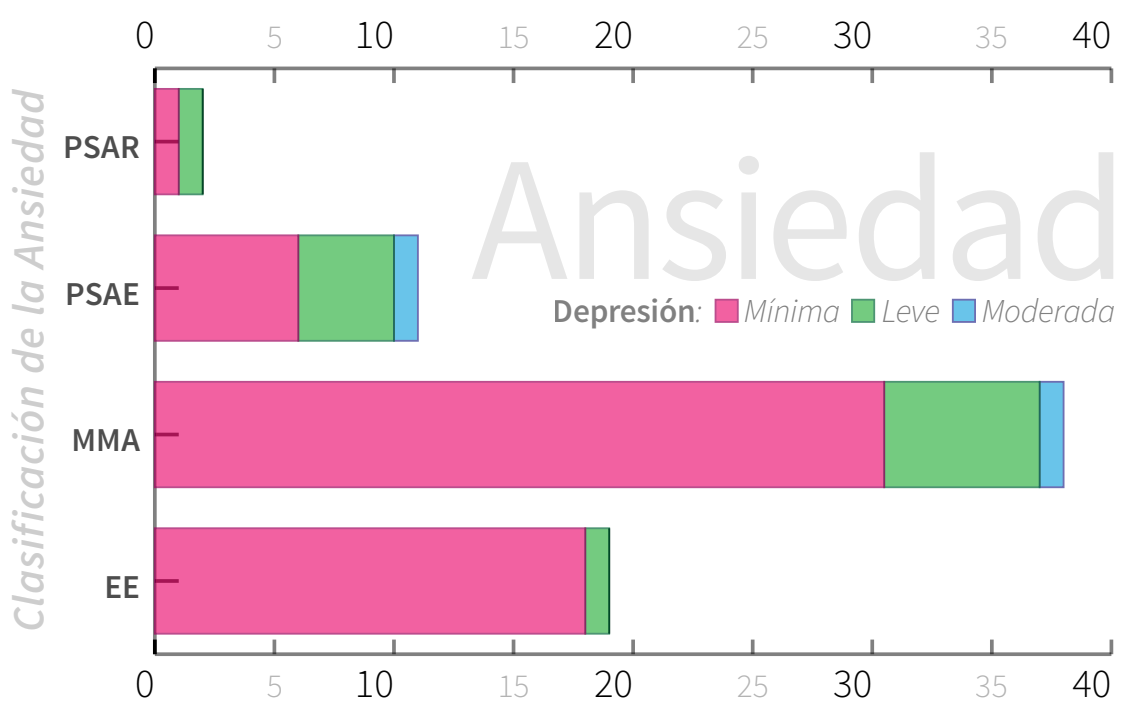

Gráfica 2 Boxplot de Ansiedad-estado decatipo en las categorías de depresión

PSAR: Presencia de síntomas de Ansiedad-Rasgo | PSAE: Presencia de síntomas de Ansiedad-Estado | MMA: Mínima Molestia de Ansiedad | EE: Emocionalmente Estable Fuente: Elaboración Propia

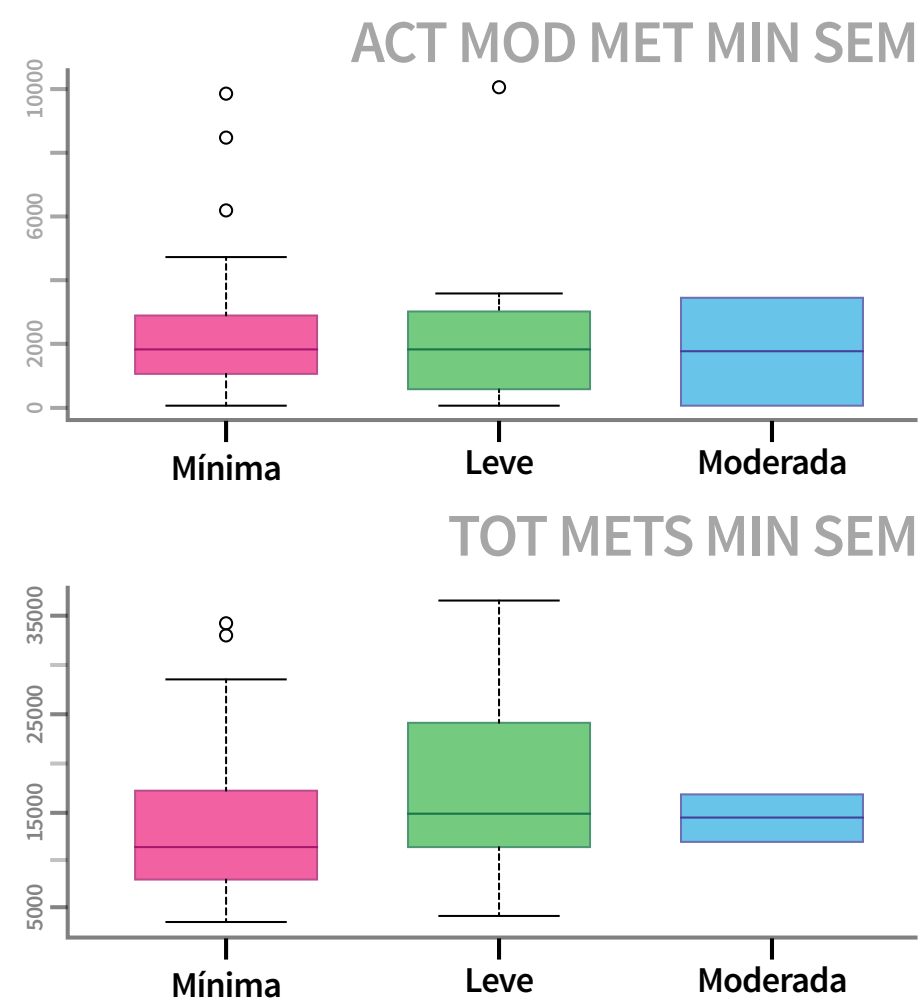




\section{Estados Emocionales y Nivel de Actividad Física}

en la Escuela Militar General José María Córdova

Tabla 1 Ansiedad-estado decatipo en las categorías de depresión

\begin{tabular}{|c|c|c|c|c|c|c|c|}
\hline \multirow[t]{2}{*}{ Variables } & \multicolumn{2}{|c|}{$\begin{array}{l}\text { Depresión } \\
\text { Mínima }\end{array}$} & \multicolumn{2}{|c|}{$\begin{array}{l}\text { Depresión } \\
\text { leve }\end{array}$} & \multicolumn{2}{|c|}{$\begin{array}{l}\text { Depresión } \\
\text { moderada }\end{array}$} & \multirow{2}{*}{$\frac{5}{\frac{0}{0}}$} \\
\hline & Med & D.E. & Med & D.E. & Med & D.E. & \\
\hline Ansiedad estado PD & 14.32 & 7.38 & 23.00 & 8.17 & 25.00 & 2.83 & 0.00 \\
\hline Ansiedad estado decatipo & 4.20 & 1.58 & 5.92 & 1.56 & 6.50 & 0.71 & 0.00 \\
\hline Ansiedad estado centil & 29.57 & 23.24 & 59.08 & 23.65 & 65.00 & 14.14 & 0.00 \\
\hline
\end{tabular}

En la Gráfica 3 (anterior) se compara la ansiedad-rasgo con las categorías de depresión, se aprecia una mediana con comportamiento similar, es decir, no hay relación entre la presencia de síntomas de ansiedad-rasgo y la presencia, en algún grado, de depresión. Este hecho se evidencia en la Tabla 2 que registra un valor p>0.05 en el cruce de estas variables.

En la Gráfica 4 se refleja un análisis bivariado que cruza la variable de depresión con los MET usando la prueba de Kruskal wallis, esta muestra un valor $p>0.05$. Esto supone que no hay una relación estadísticamente significativa entre las variables, es decir que el nivel de actividad física no tiene relación con el grado de depresión que se experimente como se evidencia también, en la tabla que se describe a continuación.

Tabla 3 Met en las categorías de ansiedad

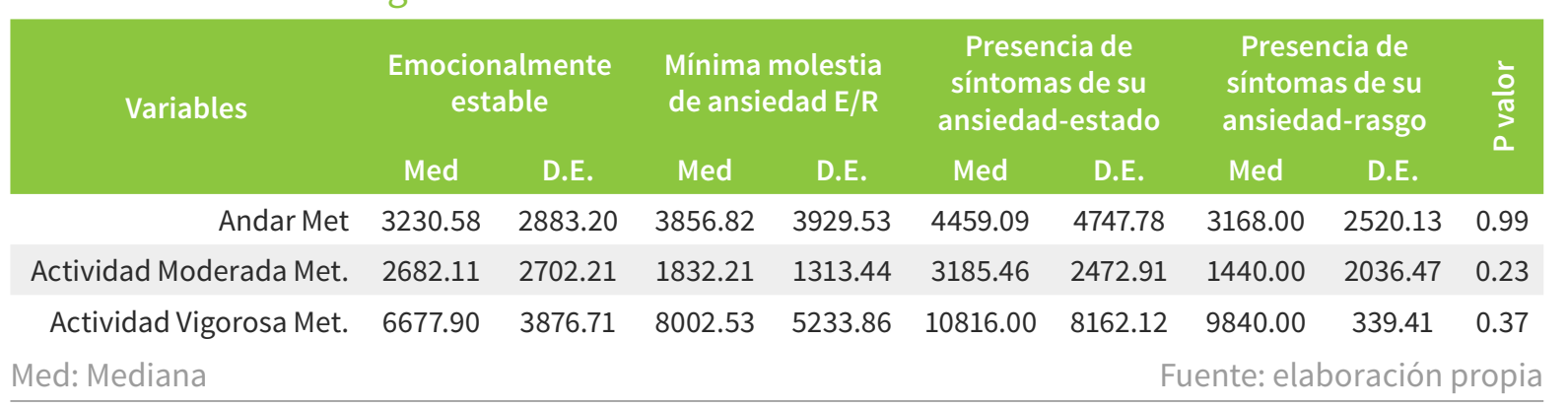

Tabla 2 Ansiedad-rasgo decatipo en las categorías de depresión

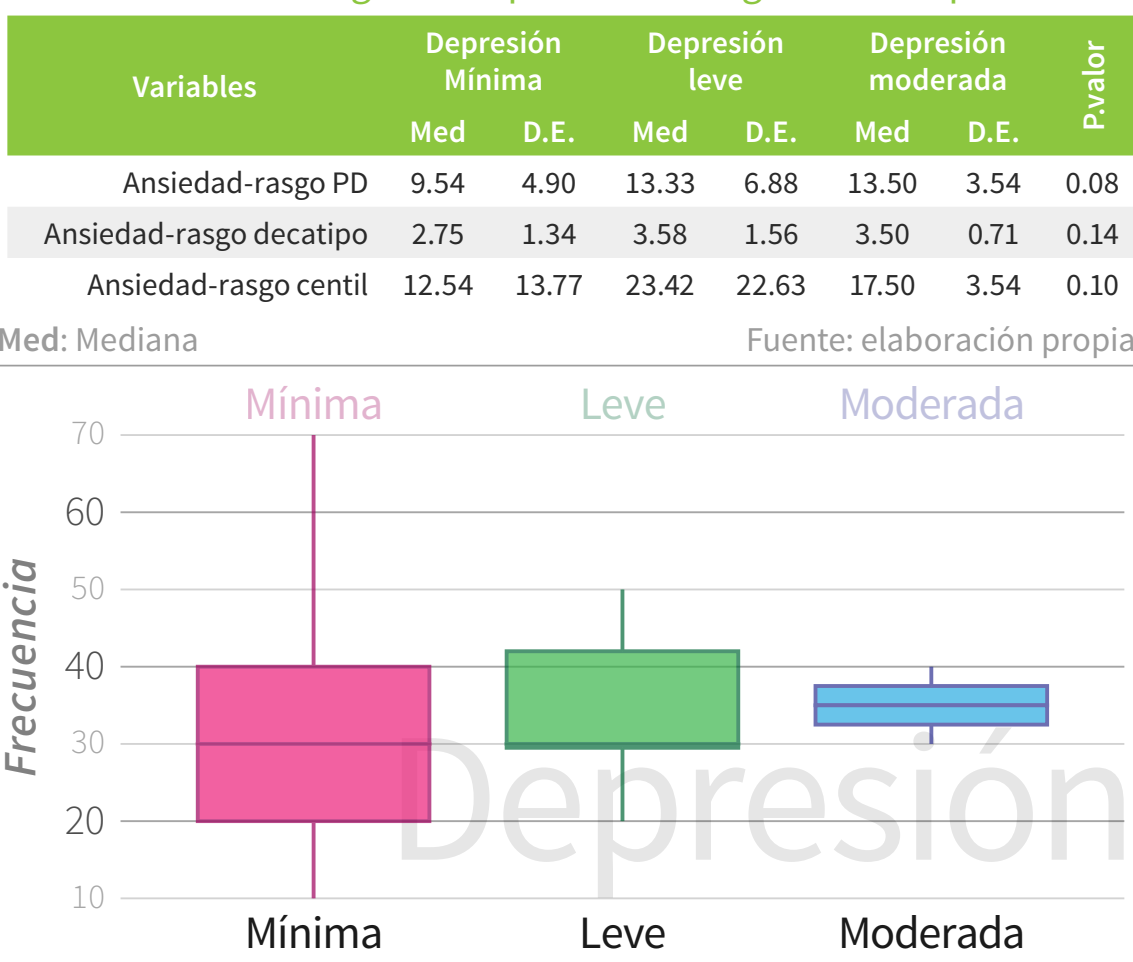

Gráfica 4 Boxplot de MET en las categorías de depresión

Fuente: elaboración propia

De manera similar, en la siguiente tabla se realiza la comparación entre las categorías de ansiedad y MET. Al llevar a cabo el análisis estadístico, no se encontraron diferencias estadísticamente significativas.

En la Tabla 3, el valor $\mathrm{p}>0.05$ indica que el nivel de actividad física no tiene relación con el grado de ansiedad que se experimente.
La Gráfica 5, en la que se analiza el IMC en cada una de las categorías de depresión, no se evidencia alguna diferencia entre las medianas, y por lo tanto no se puede hablar de influencia entre estas variables.

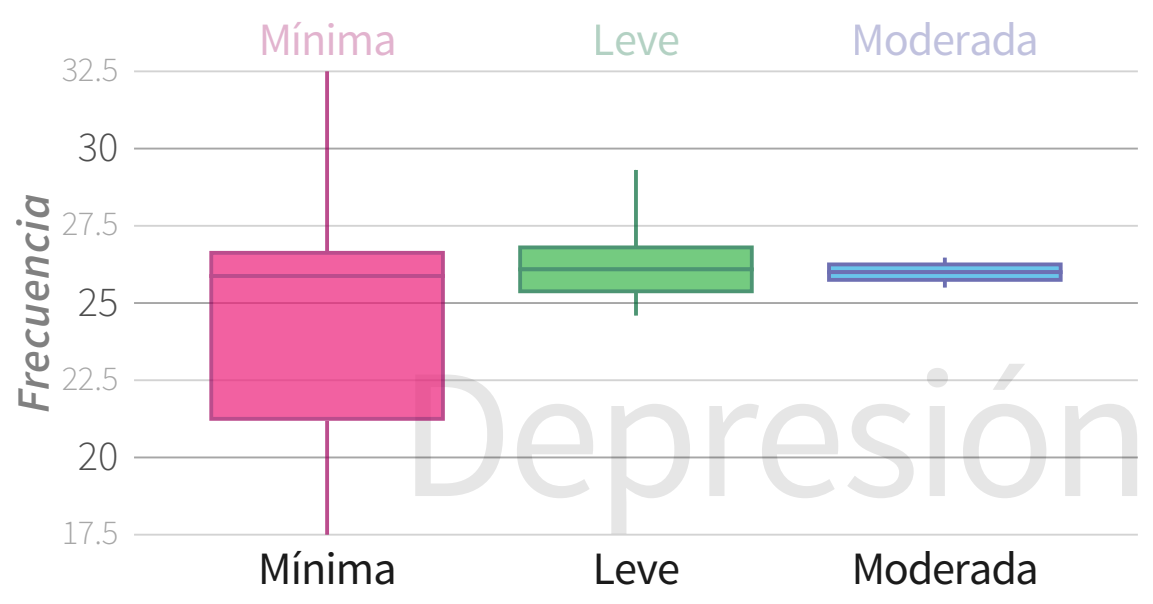

Gráfica 5 Boxplot de IMC

comparado con las categorías de depresión

Fuente: elaboración propia.

En la Gráfica 6 (sigioente) se explora la relación que existe entre las variables de ansiedad-estado y ansiedad-rasgo, entre sí y con respecto al IMC. Entre la ansiedad-estado y la ansiedad-rasgo existe un coeficiente de correlación de r2=0.52, siendo este significativo. Por otra parte, al comparar estas dos variables con el IMC no se observa alguna correlación, debido a que la ansiedad-estado es de $r 2=0.058$ y la ansiedad-rasgo es de $r 2=0.13$, con lo cual puede decirse que estas variables se comportan de manera diferente.

\section{Discusión}

Ahora bien, si se realiza un balance de los hallazgos del estudio en las variables psicológicas evaluadas, tenemos que la mayor parte de la muestra se ubica en la categoría de mínima depresión, así como en la categoría de mínima molestia de ansiedad o ansiedad media. (Villaseñor Ponce, 2010) menciona que la ansiedad leve, o moderada, puede cumplir una función favorecedora para el individuo en tanto que puede resultarle adaptativa, ya que genera un estado de alerta y tensión que puede mejorar el rendimiento. De esta manera, se entiende que el perfil psicológico de los estudiantes de la muestra en general es predominantemente funcional en estos factores.

En el estudio adelantado por Vásquez Guzmán, (2014) se encontró que la depresión y la dieta hipercalórica son factores de riesgo para el desarrollo de sobrepeso, u obesidad, y que la actividad física actuó como factor protector en población militar. Además, se destacó que la ansiedad no constituyó un factor de riesgo.

En el presente estudio, basándonos en la muestra seleccionada, no se observó relación entre la ansiedad-estado y la ansiedadrasgo, en comparación con el IMC. Pero a diferencia de los resultados encontrados en el estudio de Vásquez Guzmán (2014) no se evidenció influencia de la depresión en el IMC o viceversa, aunque estas variables de estados emocionales fueron evaluadas con instrumentos diferentes y solo se analizaron en la población con peso normal y sobrepeso.

Según el estudio desarrollado por Vásquez Guzmán (2014) la actividad física expresada en METS refleja un gasto energético considerable en la población evaluada, que también puede actuar como factor protector en tanto que no tenemos casos de obesidad. 

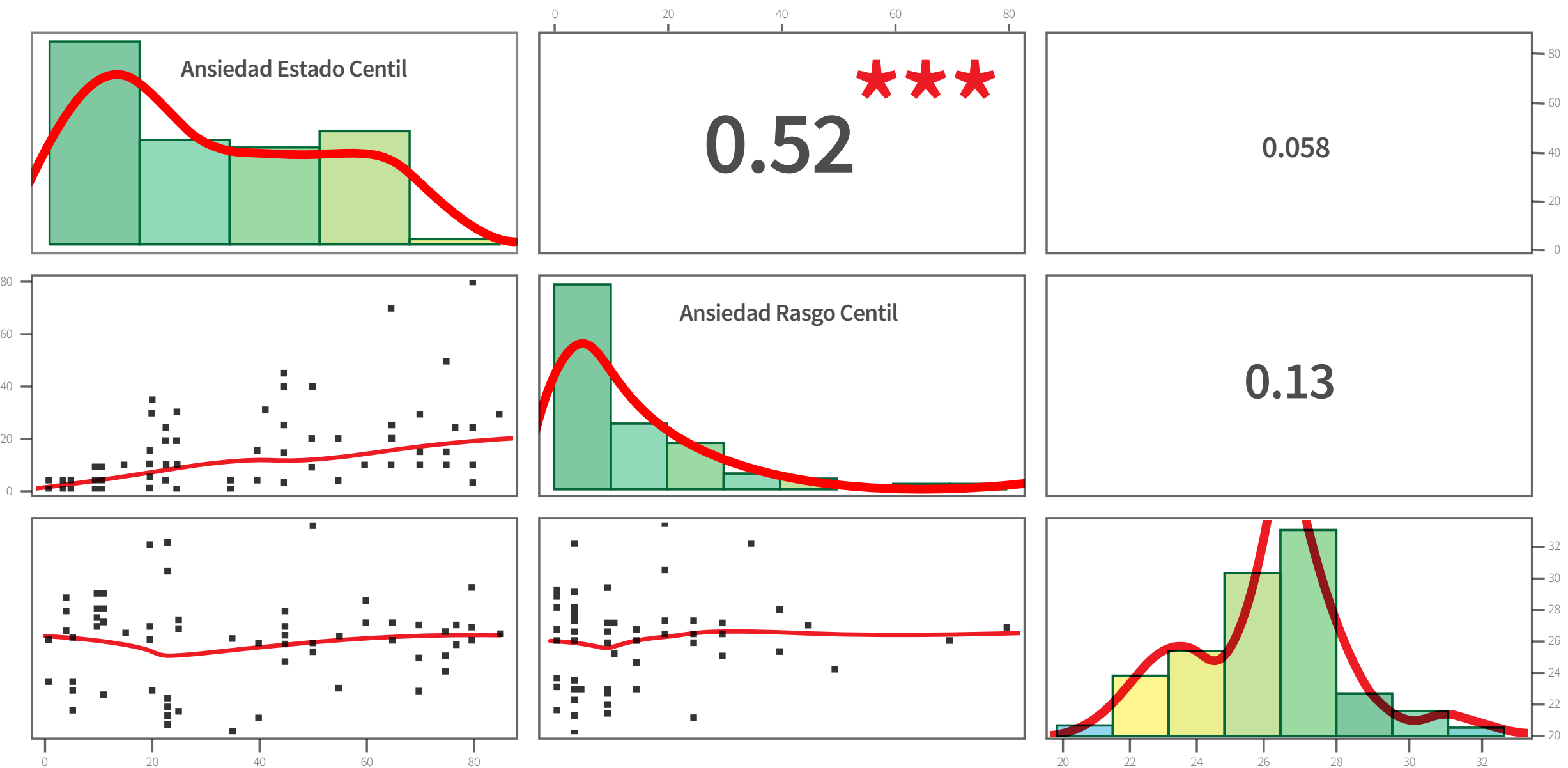

Gráfica 6 Análisis de correlación entre ansiedad-estado y rasgo y ansiedad e IMC

Fuente: elaboración propia.

Por otro lado, en un estudio realizado por Gómez, Duperly, Lucumí, Gámez y Venegas (2013) en población colombiana, encontraron que el $\mathbf{3 6 . 8 \%}$ de los sujetos son regularmente activos, mientras que en el estudio de Mahecha Matsudo, Rodrigues Matsudo y Barros Neto (2001) realizado en Sao Paulo, Brasil, demostró que un $\mathbf{5 3 . 5 \%}$ son regularmente activos. Estos resultados contrastan con los encontrados en el presente estudio en donde se halló que la actividad física obtuvo un nivel $\mathbf{3}$, es decir, que en todos los sujetos investigados se reveló un consumo calórico superior a los 5000 MET/min/sem. Probablemente estas diferencias se marcan porque el momento de aplicación de los instrumentos coincidió con la realización de las actividades con un alto grado de exigencia física, en la semana de entrenamiento específico militar realizada en el

\section{Centro Nacional de Entrenamiento del Ejército de Colombia.}

Así mismo, se analizó la clasificación de la ansiedad en todas las variables MET, lo que dio como resultado que no existe suficiente evidencia estadística para rechazar la hipótesis nula. Por lo tanto, las medianas de las cuatro categorías de ansiedad son iguales, es decir, que, en las categorías de emocionalmente estable, mínima molestia de ansiedad, presencia de síntomas de ansiedad-estado y presencia de síntomas de ansiedad-rasgo no hay ninguna diferencia según el nivel de actividad física. En el cuadro que ilustra la comparación de estas variables, parecería haber un mayor gasto energético por total METS cuando existe presencia de síntomas de ansiedad-estado, pues el valor de la mediana es mayor que las otras. Sin embargo, esta es solo una posible lectura de la tabla, y no es nada concluyente.

Ahora bien, al comparar la ansiedad y la depresión, se aprecia que en la ansiedad-estado decatipo el promedio asciende a medida que aumenta la percepción de algún grado de depresión. El comportamiento que se observa en la muestra seleccionada refleja que la variable ansiedad-estado decatipo presenta una mayor dispersión en las categorías depresión mínima y depresión leve. Por el contrario, la que tiene mayor promedio de puntaje es la variable de depresión moderada, siendo esta la de menos dispersión. Sin embargo, es posible observar que las tres categorías mantienen un comportamiento constante.

En cuanto al análisis de la ansiedad-rasgo, las medianas son estadísticamente similares: contrario a lo anterior, en la variable ansiedad-estado sí se aprecian diferencias significativas al compararla en cada una de las categorías de depresión (prueba no paramétrica Kruskalwallis, p<0.05). De esta forma, se puede inferir de acuerdo a la muestra seleccionada que, a mayor categoría de depresión, mayor puntaje de ansiedad-estado.

Finalmente, al comparar las otras variables del estudio se constata que el IMC, en relación con la variable MET, no muestra una diferencia significativa entre variables. Por otro lado, al indagar en la relación que existe entre las variables de ansiedad-estado y ansiedad-rasgo centil con respecto al IMC, se observa que entre la ansiedad-estado y ansiedad-rasgo existe un coeficiente de correlación de $r 2=0.52$, siendo este significativo, mientras que estas dos variables de ansiedad comparadas respecto del IMC, no presentan alguna correlación. Es decir que estas variables se comportan de manera diferente. Así mismo, si se compara el IMC con las categorías de depresión, se podría inferir que la depresión influye en el grado de IMC, sin embargo, en la muestra escogida, no se observa un comportamiento claro que evidencie esta hipótesis.

\section{Conclusiones}

Llegados a este punto, se evidencia que, en la comparación de las variables de estados emocionales y de actividad física del presente estudio, la variable de actividad física vigorosa comparada con las categorías de depresión parecía inicialmente reflejar alguna diferencia importante. Sin embargo, al realizar pruebas no paramétricas, se constató que en todas las variables MET, estas fueron estadísticamente iguales para las categorías de depresión. Lo anterior indica que no hay influencia entre estas y el nivel de actividad física.

Es importante destacar que los grupos poblacionales de peso normal y sobrepeso que se analizaron en este estudio se parecen en sus características, y es posible que por esto no se hayan evidenciado diferencias significativas en las variables estudiadas. 


\section{Estados Emocionales y Nivel de Actividad Física}

en la Escuela Militar General José María Córdova

Para concluir, se recomienda que es importante indagar en futuras investigaciones la conducta alimentaria de los estudiantes, ya que es un factor que posiblemente es relevante en el IMC de la muestra. Igualmente, en la población podrían incluirse sujetos con bajo peso y obesidad, y así aumentar el tamaño de la muestra.

\section{Agradecimientos}

El presente estudio no presenta conflictos de intereses y fue financiado en su totalidad por la Escuela Militar de Cadetes General José María Córdova.

\section{Referencias}

Borba Neves, E. (2008). Prevalência de sobrepeso e obesidade em militares do exército brasileiro: associação com a hipertensão arterial. Ciência \& Saúde Coletiva, 13(5), 1661-1668. doi: http://dx.doi.org/10.1590/S141381232008000500029

Bustamante, D., Sbarra, J., Yuma, F., Laborda, R., Mendoza, J., Pereyra, J. C., \& Alvarez, V. (2011). Antropometría y composición corporal búsqueda del mejor indicador de sobrepeso en el cadete naval, Escuela Naval Militar. Archivos Militares, 8, 55 - 68. Obtenido de http://www.inun.edu.ar/menues/ investigacion/revista/REVISTA\%20DIGITAL\%20DEL\%20INUN.pdf\#page=57

Castañeda Tovar, S. M., \& Caiaffa Bermúdez, N. S. (2015). Relación entre la composición corporal y el rendimiento físico en la Escuela Militar José María Córdova. Revista Científica General José María Córdova, 13(1), 257270. doi: http://dx.doi.org/10.21830/19006586.26

Córdoba, J. F. (2011). Revisión bibliográfica sobre las características sociodemográficas y repercusiones de la depresión en el trabajador. Medicina y Seguridad del Trabajo, 174-187.

Craig, C. L., Marshall, A. L., Sjöström, M., Bauman, A. E., Booth, M. L., Ainsworth, B. E., .. . Oja, P. (2003). International physical activity questionnaire: 12 -country reliability and validity. Medicine \& Science in Sports \& Exercise, 35(8), 1381-1395. doi: https://doi.org/10.1249/01.MSS.0000078924.61453.FB

Gómez, L. F., Duperly, J., Lucumí, D. I., Gámez, R., \& Venegas, A. S. (2013). Nivel de actividad física global en la población adulta de Bogotá: Prevalencia y factores asociados. Gaceta Sanitaria, 19(3), 206-213. Obtenido de http:// scielo.isciii.es/scielo.php?script=sci arttext\&pid=S0213-91112005000300005

Heyward, V. H. (1996). Evaluación y prescripción del ejercicio (ilustrada ed., Vol. 1). Barcelona: Paidotribo.
ISAK. (2001). International Standards for Anthropometric Assessment (1 ed., Vol. 1). Holbrooks (au): International Society for the Advancement of Kinanthropometry. Obtenido de http://www.ceap.br/material/ MAT17032011184632.pdf

Loaiza, O., \& Posada, J. L. (2016). Psicología militar: conceptualización e investigaciones contemporáneas. Revista Latinoamericana de Ciencia Psicológica - Psiencia, 8(2). doi: http://dx.doi.org/10.5872/psiencia/8.2.71

Mahecha Matsudo, S., Rodrigues Matsudo, V. K., \& Barros Neto, T. L. (2001). Atividade física e envelhecimento: aspectos epidemiológicos. Revista Brasileira de Medicina do Esporte, 7(1), 1-13. doi: http://dx.doi.org/10.1590/ S1517-86922001000100002

Pérez Hidalgo, A. M., \& Rodríguez González, J. M. (2011). Análisis del estado emocional de una unidad del ejército español en zona de operaciones. Sanidad Militar, 67(2), 71-77. Obtenido de: http://scielo.isciii.es/scielo. php?script=sci arttext\&pid=S1887-85712011000200002

Sánchez Daza, L. J., Herrera González, Y. E., \& Daza Mora, M. C. (2012). Evaluación del riesgo cardiovascular usando la escala de Framingham en aviadores militares del Ejército Colombiano. Trabajo de Grado Especialización, Universidad del Rosario, Escuela de Medicina y Ciencias de la Salud, Bogotá D.C. Obtenido de http://repository.urosario.edu.co/ handle/10336/2828

Sanz Fernández, J., Vázquez Valverde, C., \& Luis Perdigón, A. (2003). Adaptación española del Inventario para la Depresión de Beck-II (BDI-II): 2. Propiedades psicométricas en población general. Clínica y salud, 14(3) 249-280. Obtenido de http://wwW.copmadrid.org/web/articulos/2003143/ clinicaysalud

Sarason, B. G., \& Sarason, I. G. (2006). Psicopatologia. Psicologia Anormal. El problema de la conducta inadaptada (Vol. 1). Mexico: Pearson Education.

Spielberger, C. D., Gorsuch, R. L., \& Lushene, R. E. (2011). STAI. Cuestionario de Ansiedad-estado-Rasgo (8a ed., Vol. 1 Manual). (G. Buela Casal, A. Guillén Riquelme, \& N. Seisdedos Cubero, Edits.) Madrid (es): TEA Ediciones.

Vásquez Guzmán, M. A. (2014). Hábitos alimentarios, actividad física, y binomio ansiedad/depresión, como factores de riesgo asociados con el desarrollo de obesidad en población militar. Sanidad Militar, 70(1), 25 - 29. doi: http://dx.doi.org/10.4321/S1887-85712014000100004

Villaseñor Ponce, M. (2010). Correlación entre ansiedad y bienestar psicológico en estudiantes que ingresan a la universidad. (J. Lucero, \& E. Eduardo, Edits.) Revista Mexicana de Psicología Educativa, 1(1), 42. Obtenido de http://www.psicol.unam.mx/silviamacotela/Pdfs/RMPE_VIN1_A5.pdf

Citar como: 